\title{
PRÁTICAS EDUCATIVAS E DOCÊNCIA: EM BUSCA DO ENSINO CRÍTICO
}

\author{
${ }^{1}$ Gildoberg Nunes da Silva, Universidade Federal de Campina Grande - UFCG, \\ bergnunes22@gmail.com \\ ${ }^{1}$ Danielli Cristina de Lima Silva, Universidade Federal da Paraíba - UFPB \\ limaanacrisdani@gmail.com \\ ${ }^{2}$ Henrique Miguel de Lima Silva, Universidade Federal da Paraíba - UFPB, \\ henrique.miguel.91@gmail.com
}

\begin{abstract}
RESUMO
Este trabalho trata se de uma pesquisa bibliográfica onde se buscou uma análise de práticas educativas que compõem a educação de alunos, através de pesquisa bibliográfica, fundamentados em Brasil (1998, 2012) Freire (1996,1996), Ferraço (2005), dentre outros, para constatação da hipótese levantada com a discussão de que o aluo adquire um percentual maior e mais eficiente de conhecimento e saberes através de práticas realizadas por docentes qualificados capazes de visualizar a realidade acadêmica e social do aluno para garantir uma aprendizagem em plenitude com o docente sendo capaz de aprender à ensinar flexibilizando e/ou adaptando os conteúdos programáticos às realidades socioculturais dos meios em ambos (docente e discente), estão inseridos. Partimos da assertiva de que a formação, quando crítica, possivilita a diminuição dos problemas sociais, bem como contribui diretamente para a autonomia dos envolvidos do processo, diminuindo, assim, a formação para reprodução. Dessa maneira, o docente desempenha o papel fundamental de orientar as aprendizagem, enquanto práticas críticas, situadas e contextualizadas às necessidades dos educandos.
\end{abstract}

Palavras-chave: Alunos, educação, práticas.

\section{EDUCATIONAL PRACTICES AND TEACHING: IN SEARCH OF CRITICAL TEACHING}

\section{SUMMARY}

This work is about a bibliographical research where an analysis of educational practices that compose the education of students through bibliographical research, based on brazil (1998, 2012), freire $(1996,1996)$, ferraço (2005), among others , to verify the hypothesis raised with the discussion that the student acquires a greater and more efficient percentage of knowledge and knowledge through practices performed by qualified teachers able to visualize the academic and social reality of the student to ensure a full learning with the teacher being able to learn to teach flexible and / or adapting the programmatic contents to the sociocultural realities of the media in both (teacher and student), are inserted. We start from the assertion that training, when critical, allows the reduction of social problems, as well as contributes 
directly to the autonomy of those involved in the process, thus reducing training for reproduction. In this way, the teacher plays the fundamental role of guiding the learning, as critical practices, located and contextualized to the needs of learners.

KEYWORDS: Students, Education, Practices.

PRÁCTICAS EDUCATIVAS Y DOCENCIA: EN BUSCA DE LA ENSEÑANZA CRÍTICA

\section{RESUMEN}

Este trabajo se trata de una investigación bibliográfica donde se buscó un análisis de prácticas educativas que componen la educación de alumnos, a través de investigación bibliográfica, fundamentados en Brasil (1998, 2012) Freire (1996,1996), Ferraço (2005), entre otros Para constatar la hipótesis planteada con la discusión de que el aluo adquiere un porcentaje mayor y más eficiente de conocimiento y saber a través de prácticas realizadas por docentes cualificados capaces de visualizar la realidad académica y social del alumno para garantizar un aprendizaje en plenitud con el docente Siendo capaz de aprender a enseñar flexibilizando y / o adaptando los contenidos programáticos a las realidades socioculturales de los medios en ambos (docente y discente), están insertados. Partimos de la asertiva de que la formación, cuando crítica, posibilita la disminución de los problemas sociales, así como contribuye directamente a la autonomía de los involucrados del proceso, disminuyendo así la formación para la reproducción. De esta manera, el docente desempeña el papel fundamental de orientar el aprendizaje, como prácticas críticas, situadas y contextualizadas a las necesidades de los educandos.

Palabras clave: Alumnos, educación, prácticas.

\section{INTRODUÇÃO}

As práticas na educação deveria ter um retorno que remete aprendizagem para formação de conhecimentos dos alunos onde as leituras e teorias que lhes são oportumas dentro e fora de uma sala de aula dão origem à uma boa base para que o mesmo avançe na escrita e letramento que são considerados pilares centrais na educação e que depende do professor como profissional.

Segundo freire (1997), o professor, além de ensinar, aprende; e o aluno, além de aprender, ensina. Freire criticava a ideia de que ensinar é transmissão de conhecimento, uma vez que ao professor cabe a tarefa de possibilitar a criação ou a produção do conhecimento e 
não da sua imposição. Assim, o professor da educação básica deve buscar todas as estratégias possíveis para trabalhar o aprendizado de seu discente e de forma atrativa, pois neste período há uma desmotivação muito grande por parte dos alunos para frequentar regularmente a escola, como veremos na análise de nossa pesquisa.

[...] Os alunos constroem significados a partir de múltiplas e complexas interações. Cada aluno é sujeito de seu processo de aprendizagem, enquanto o professor é o mediador na interação dos alunos com os objetos de conhecimento; o processo de aprendizagem compreende também a interação dos alunos entre si, essencial à socialização. (BRASIL, 1997, P.58).

Diante do exposto, compreende-se que:

A identidade (profissional) se constrói simultaneamente no juízo que o indivíduo faz de si próprio, tendo como referência os seus julgamentos sobre os outros, os julgamentos dos outros sobre ele próprio, como também o contexto social em que está inserido. (ERIKSON, 1976, P.24).

Consequentemente, é incontestável que a afetividade é um mecanismo de sustentabilidade de grande importância para o processo de ensino e aprendizagem, visto que, como podemos perceber em vygostky (1988), o processo de aprendizagem tende a ser complexo, evolutivo, com muitos matizes contextuais, que dependem vitalmente da interação social e intenso intercâmbio de significados.

A prática pedagógica atinge um nível de complexidade, porque o docente ver-se envolvido em diversas atividades em conteúdos. O ensino atualmente exige, uma ampla gama de conhecimentos dispostos em conteúdos que são importantes para a formação do educando, é uma extrema responsabilidade selecioná-los de modo que o educando possa conhecê-lo de forma satisfatória a fim de prepara-se para sua formação (PERRENOUD, 2002, P. $11)$.

É preciso acrescentar que:

O papel do novo professor é o de usar a perspectiva de como se dar a aprendizagem, para que usando a ferramenta dos conteúdos postos pelo ambiente e pelo meio social, estimule as diferentes inteligências de seus 
alunos e os leva a se tornarem aptos a resolver ou quem sabe, criar produtos válidos para seu tempo e sua cultura. (ANTUNES, 1999, P.98)

\section{METODOLOGIA}

Trata-se uma pesquisa de caráter descritivo. Assim, tomamos como referência as orientações de Gil (2002), e bibliográfica por trata-se do levantamento, seleção e documentação que delibera sobre o assunto.

Para ser feita essa pesquisa foi utilizada a pesquisa bibliográfica de teóricos que são considerados bases fortes na formulação de hipóteses que deram ciência para as experiências serem fundamentos que consideram-se de suma importância por fato de no trabalho docente com o alunado ter um envolvimento com a escrita e o letramento.

Nossa pesquisa foi fundamentada nos teóricos seguintes: Albuquerque; Morais (2010) Alegria (2001), Franco (2012), Freire (1996), Gomide (2001) Imbert (2003), Oliveira (2007), Pacheco (2004), dentre outros que contribuíram para a fundamentação teórica.

\section{A FORMAÇÃO PARA AUTONOMIA: PRINCÍPIOS EDUCATIVOS}

A educação, além de ser um ato político e um posicionamento ideológico contra uma sociedade segregadora de classes e etnias, busca democratizar a sociedade tendo como base os conhecimentos científicos. Para isto, faz-se necessário a formação dos docentes, tanto nos cursos de graduação e pós-graduação, como as formações continuadas. Sabe-se ainda que:

A educação continuada de professores envolve a perspectiva da formação pessoal e do autoconhecimento. Enfoca pela necessidade de interação entre contextos diversos e a necessidade de entender o mundo e a sua inserção profissional. Neste processo de formação, a valorização do potencial dos professores de sua criatividade e expressividade no processo de ensinar e aprender (TOSCHI, 2001, P.81).

Neste sentido, faz-se necessário:

É preciso estabelecer as condições não apenas quantitativas, mas também qualitativas de um tempo educativo que [..] O tempo escolar é um tempo 
social, uma construção cultural e pedagógica, um "fato cultural" e, portanto, deve ser flexível, singular e adaptado em função das características de cada contexto escolar. (GOMES, 2004, P. 48).

Um tema indispensável consiste na formação voltada para cada área do saber científico. Infelizmente, ainda temos a lamentável prática de docentes exercendo funções para as quais não tem formação especifica.

Sabe-se ainda que a:

Necessidade de formação especifica e profissionalizante para o educador, em oposição a um histórico de voluntariado, ate hoje incentivado por campanhas de órgãos oficiais, em um fragrante descomprometimento com a garantia do direito a educação básica de qualidade, que não se pode negar ao cidadão, numa sociedade que se quer democrática (BARCELOS, 2006, P.134).

Ainda Em Se Tratando Desse Processo Formativo Crítico, Acrescenta-Se Que:

Isso implica: construção de espaços para reflexão crítica, flexibilização e criação de canais de informação nas escolas, alianças e apoios entre os profissionais e implementação de políticas públicas de valorização e formação docente. portanto, precisamos conceber a formação continuada dos educadores como elemento crucial para a (re)construção da instituição escolar. (ALMEIDA, 2004, p. 244)

Em consonância com o que foi dito anteriormente, Almeida e Martins (2009, p. 17) aludem que:

Acreditamos que as boas práticas pedagógicas sejam apropriadas a todos os alunos, inclusive àqueles com necessidades educacionais especiais. (...) Em alguns momentos e contextos, esses alunos podem precisar de flexibilizações mais significativas ou de atendimentos mais específicos. Um currículo, que tenha como princípio a diferença deverá considerar todas essas situações e vivências.

Outro ponto que merece destaque tem sido nossas legislações educacionais que, na maioria das vezes, são insuficientes e não tem considerado com a devida relevância os 
procedimentos necessários para uma educação de qualidade e baseada na perspectiva crítica de ensino.

Diante disso:

Políticas públicas brasileira no que diz respeito à educação têm algumas fragilidades que impedem o seu desempenho: tem como base o interesse internacional; a falta de clareza em muitas de suas leis e decretos; a falta de participação dos profissionais de ensino nas discussões de políticas públicas; a dificuldade dos professores em mudar seus procedimentos metodológicos; a falta de qualificação dos profissionais; etc., neste contexto surge formas em sua maioria artificiais na abordagem dos conteúdos. (SILVA FILHO, 2012, p. 42)

Pimenta (1997, p. 6), acrescenta que faz-se necessário adotarmos um posicionamento crítico de que o processo formativo não se resume a mera transmissão de conhecimento, quando na verdade, precisamos nos posicionar quanto a compreensão do ensino como realidade social e, que desenvolva a prática da autorreflexão e através da mesma buscar transformarem os seus saberes-fazeres docentes, num processo contínuo de construção de suas identidades como professores.

Caracteriza-se como um novo princípio educacional, cujo conceito fundamental defende a heterogeneidade na classe escola, como situação provocadora de interações entre crianças com situações pessoais as mais diversas e busca-se uma pedagogia que se dilate frente às diferenças dos alunos. (BEYER, 2006, p. 73)

Somente quando for levada em consideração a realidade social do docente e em que a escola encontra-se inserida, bem como as necessidade das famílias dessa região, bem como os anseios dos educandos é que teremos um processo educativo em sua plenitude. É necessário educar para autonomia e interação social, prática que passa desapercebida pela sublimação da classe docente aos lamentáveis conflitos cotidianos do nosso trabalho.

\section{CONSIDERAÇÕES FINAIS}

Somente um ensino prático, crítico e que trabalhe com os problemas sociais de forma cotidiana e reflexiva é que atingiremos o sucesso desejado nas práticas docentes, deve haver envolvimento de todos os envolvidos No contexto do docente assim faz-se indispensável 
considerar a realidade sociocultural da escola, das famílias e dos educandos, uma vez que a educação deve resolver os conflitos sociais com base no conhecimento científico.

Ainda em se tratando da docência, faz-se necessário que o docente adeque sua didática, bem como sua metodologia de ensino para oferecer autonomia, algo que só é possível quando o aluno é co-responsável pelo processo de formação de conceitos, saberes e práticas.

Dessa maneira, espera-se que as práticas de docência considerem como primordial a superação das segregações sociais, oferecendo recursos necessários para transformação social.

$\mathrm{Na}$ pesquisa constatou que para o processo de educação dos alunos precisa-se de docentes com qualificação, que para as atividades em sala de aula, o docente necessita de tempos para planejar essas atividades e promover o encontro das mesmas com seu alunado e encontrou-se que, através das ideias partidas do trabalho docente com práticas educativas deva privilegiar o cotidiano escolar e os saberes úteis que são vivenciados na realidade atual do docente com o aluno, na troca de conhecimentos. 


\section{REFERÊNCIAS}

AINSCOW, MEL; PORTER, GORDON; WANG, MARGARET. CAMINHOS PARA AS ESCOLAS INCLUSIVAS. LISBOA: INSTITUTO DE INOVAÇÃO EDUCACIONAL, 1997. [TEXTOS ORIGINAIS EM INGLÊS, APRESENTADOS EM SALAMANCA, 1994.]

ALMEIDA, MARIANGELA LIMA DE. FORMAÇÃO CONTINUADA COMO PROCESSO CRÍTICO-REFLEXIVO COLABORATIVO: POSSIBILIDADES DE CONSTRUÇÃO DE UMA PRÁTICA INCLUSIVA. 2004. 263 F. DISSERTAÇÃO (MESTRADO EM EDUCAÇÃO) - PROGRAMA DE PÓS-GRADUAÇÃO EM EDUCAÇÃO, UNIVERSIDADE FEDERAL DO ESPÍRITO SANTO, VITÓRIA, 2004.

ALMEIDA, MARIANGELA LIMA DE; MARTINS, INES DE OLIVEIRA RAMOS. PRÁTICA PEDAGÓGICA INCLUSIVA: A DIFERENÇA COMO POSSIBILIDADE. VITÓRIA, ES: GM, 2009. P. 17.

ANTUNES, IRANDÉ. LÍNGUA, TEXTO E ENSINO - OUTRA ESCOLA POSSÍVEL. SÃO PAULO, PARÁBOLA, 2009.

BARCELOS, VALDO. FORMAÇÃO DE PROFESSORES PARA A EDUCAÇÃO DE JOVENS E ADULTOS. PETRÓPOLIS, VOZES, 2006.

BRASIL, SECRETARIA DE EDUCAÇÃO FUNDAMENTAL. PARÂMETROS CURRICULARES NACIONAIS: HISTÓRIA, GEOGRAFIA. - BRASÍLIA: MEC/SEF, 1997.

BRASIL, LEI DE DIRETRIZES E BASES DA EDUCAÇÃO NACIONAL. Nº 9.394, 20 DE DEZEMBRO DE 1996. BRASÍLIA, DF, MEC, 1996. IBGE,2007.DISPONÍVEL EM: HTTP://WWW.IBGE.GOV.BR/HOME/PRESIDENCIA/NOTICIAS/NOTICIA_ VISUALIZA.PHP?ID_NOTICIA=987 ACESSADO EM ABRIL DE 2012.

DUARTE, MARTA. ALFABETIZAÇÃO DE ADULTOS. PORTO ALEGRE, ARTMED, 1998.

ERIKSON, E. IDENTIDADE, JUVENTUDE E CRISE. RIO DE JANEIRO: ZAHAR, 1976.

FREIRE, PAULO. PEDAGOGIA DO OPRIMIDO. 41. ED. RIO DE JANEIRO: PAZ E TERRA. 1996. 
FREIRE, P. PEDAGOGIA DA AUTONOMIA: SABERES NECESSÁRIOS À PRÁTICA EDUCATIVA. SÃO PAULO, PAZ E TERRA. 31 $1^{\text {a }}$ ED. 1997.

FERRAÇO, C. E. CURRÍCULO, FORMAÇÃO CONTINUADA DE PROFESSORES E COTIDIANO ESCOLAR: FRAGMENTOS DE COMPLEXIDADE DAS REDES VIVAS. IN: FERRAÇO, CARLOS EDUARDO (ORG.). COTIDIANO ESCOLAR, FORMAÇÃO DE PROFESSORES (AS) E CURRÍCULO. SÃo PAULO, CORTEZ, 2005.

GADOTTI, MOACIR. BONITEZA DE UM SONHO: ENSINAR E APRENDER COM SENTIDO. NOVO HAMBURGO: FEEVALE, 2003. 80 P.

GIL-PÉREZ, D.; CARVALHO, A. M. DE C. FORMAÇÃO DE PROFESSORES DE CIÊNCIAS: TENDÊNCIAS E INOVAÇÕES.8 ED. SÃO PAULO: CORTEZ, 2002. 120 P.

GOMES, C. A.. A EDUCAÇÃo EM NOVAS PERSPECTIVAS SOCIOLÓGICAS. $4^{a}$ ED. SÃO PAULO: ED. PEDAGÓGICA E UNIVERSITÁRIA, 2004.

HOFFMANN, J. AVALIAÇÃO MITO \& DESAFIO: UMA PERSPECTIVA CONSTRUTIVISTA. 26. ED. PORTO ALEGRE: MEDIAÇÃO, 2000.

LibÂNeO, José C. PEDAgogia E PEDAgogos Para QUÊ? 6 ED.SÃo PAULO:CORTEZ,2002.

PIAGET, J. SEIS ESTUDOS DE PSICOLOGIA. FORENSE, 1967. 
